\title{
Zurück an die Uni?
}

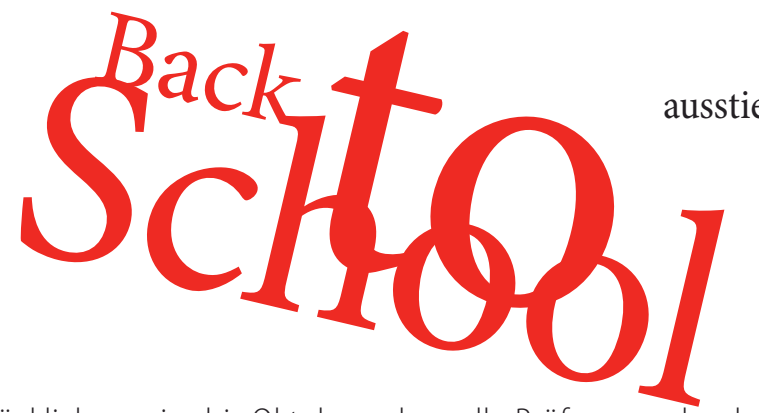

Da ich mein Examen ja bereits im Sommer 2009 begonnen und glücklicherweise bis Oktober schon alle Prüfungen abgelegt hatte, hoffte ich noch vor Weihnachten meine Approbationsurkunde in Händen halten zu können. Nach fast einem halben Jahr der Abstinenz vom Behandeln könnte ich so bald wieder den Bohrer schwingen!

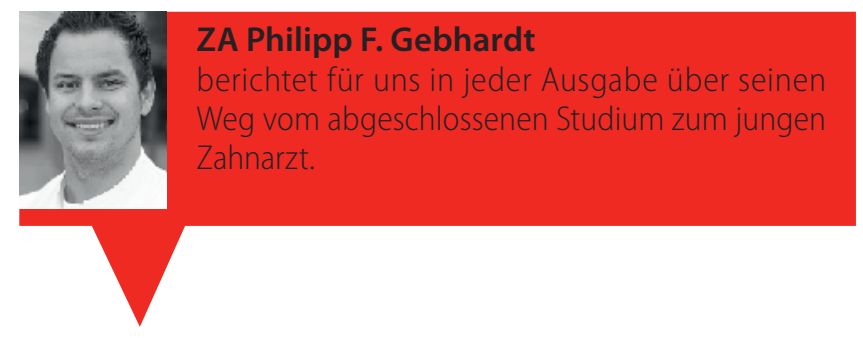

\section{Im Behörden-Labyrinth}

Diese Rechnung hatte ich allerdings ohne die offensichtlich unerlässlichen, aber zeitraubenden und vor allem undurchsichtigen, administrativen Hürden gemacht. Denn die galt es noch zu nehmen, bevor der erste Patient behandelt werden durfte:

Wesentlich für den Zulassungsantrag bei KZV, ZÄK und der Meldung beim AVW (Altersversorgungswerk) ist nämlich die bereits erwähnte Approbationsurkunde, die ich natürlich nicht rechtzeitig zugeschickt bekommen hatte. Wirklich problematisch war dies für mich aber eigentlich nur, weil ich darauf angewiesen war, ab 01. Januar 2010 eine Vollanstellung über 12 Monate nachzuweisen, um 2011 meine schon unterschriebene Stelle im „Mundwerk“ in Berlin anzutreten. Durch die Unterstützung meines derzeitigen Praxisteams, gepaart mit Frust und Mitleid der zuständigen Sachbearbeiter, konnte ich dann aber doch noch alle fehlenden Schreiben nachreichen und pünktlich am 02.01.2010 meinen ersten Dienst antreten.

Nachdem ich nun immer mehr in den Praxisalltag eingestiegen war, vermehrten sich mit der wachsenden Erfahrung auch die Einträge im Bestellbuch. Die anfängliche Skepsis einiger Patienten gegenüber einem neuen Assistenzzahnarzt legte sich rasch. Dies lag nicht zuletzt an dem sowohl sympathischen als auch eingespieltem Praxisteam, mit dem viele Situationen souveräner zu meistern sind, als es die Behandlungen im Studium vermuten lassen.

\section{Unverhofft kommt oft}

Froh alle bisherigen Hindernisse erfolgreich genommen zu haben, dauerte es nur wenige Tage bis mir das Thema „Versicherungen“ vor die Füße fiel. Nach zahlreichen Versuchen aus dem reichhaltigen Pool der Versicherungsanbieter etwas Zutreffendes, Sinnvolles, Erforderliches und nicht zuletzt auch finanziell Attraktives herauszufinden, gab ich meine Eigeninitiative auf. Statt dessen wandte ich mich an einen Versicherungsmakler, der mir von zahlreichen Kollegen weiterempfohlen wurde und sich als sehr gut herausstellte.

Nachdem im Februar auch endlich meine Wohnung in Heidelberg ausgeräumt und übergeben war, blieb nun endlich Zeit die letzten Korrekturen an meiner Doktorarbeit abzuarbeiten um sie nach 3 Jahren endlich in Druck geben zu können. Genau da erhielt ich einen Anruf aus der Charité: „Herr Gebhardt, Sie hatten sich doch bei uns beworben.“, „Richtig, das war während des Examens..... Die Neugier siegte und so fand ich mich zwei Wochen später zu einem Vorstellungsgespräch beim Chefarzt der Zahnerhaltung und Parodontologie ein.

\section{Impressum}

Eigentümer \& Copyright $\odot$ Springer-Verlag 2010, Springer Medizin c/o Springer-Verlag GmbH, Tiergartenstr. 17, 69121 Heidelberg, Tel. +49 6221/487-0, www.springer.de. Springer Medizin ist ein Teil der Fachverlagsgruppe Springer Science+Business Media Geschäftsführung Springer Medizin: Harm van Maanen (Executive Vice President), Dr. Dirk Einecke, Ulrich Huber, Stephan Kröck, Dr. Esther Wieland, Matthias Wissel Leitung Zahnmedizin: Dr. Nataša Djordjević (v.i.S.d.P., Tel. -8445, natasa.djordjevic@springer.com)

Redaktion "der junge zahnarzt“: Susanne Redeker (Tel. -8980, susanne.redeker@springer.com), Sabrina Gabriel (Tel. -8170, sabrina.gabriel@springer.com) Layout: Arnulf Illing (Tel. -8952, arnulf.illing@springer.com),

Bildredaktion: Christiane Seufert

Leitung Herstellung: Johannes Buchmann

Zertifizierte Fortbildung: Dr. Paul Herrmann

Gesamtleitung Sales \& Marketing: Stephan Kröck

Anzeigen: Marita Säuberlich (Tel. -8309, Fax -68309, marita.saeuberlich@springer.com) Industriekommunikation: Anja Weisse (Tel. -8739, Fax -68739, anja.weisse@springer.com) Druck: Stürtz GmbH, Würzburg. Printed in Germany

Erscheinungsweise: 4 Ausgaben pro Jahr; Papierausgabe: ISSN 1869-5744, gedruckt auf säurefreiem Papier

Bezugspreise (unverb. Preisempfehlung inkl. 7\% deutscher MwSt. und Versand):
Vorzugspreis für persönliche Abonnenten: EUR 48,-; Einzelheftpreis: EUR 15,-- Das Abonnement kann jederzeit 2 Monate vor Ende des Bezugszeitraumes gekündigt werden. Bestellungen oder Rückfragen nimmt jede Buchhandlung oder der Verlag entgegen. Kontakt: Springer Customer Service Center GmbH, Haberstr. 7, 69126 Heidelberg, Tel. +49 6221/345-4303, Fax-4229, Leserservice@springer.com (Mo.-Fr. 8.00 Uhr bis 20.00 Uhr) Copyright \& allgemeine Hinweise: Die Zeitschrift sowie alle in ihr enthaltenen einzelnen Beiträge und Abbildungen sind urheberrechtlich geschützt. Jede Verwertung, die nicht ausdrücklich vom Urheberrechtsgesetz zugelassen ist, bedarf der vorherigen schriftlichen Zustimmung des Verlags. Das gilt insbesondere für Vervielfältigungen, Bearbeitungen, Übersetzungen, Mikroverfilmungen und die Einspeicherung und Verarbeitung in elektronischen Systemen.

Gezeichnete Artikel geben nicht unbedingt die Meinung der Redaktion wieder. Autoren können unter bestimmten Voraussetzungen an der Ausschüttung der Bibliotheksund Fotokopietantiemen teilnehmen. Einzelheiten bei VG WORT, Abt. Wissenschaft, Goethestr. 49, 80336 München.

Angaben über Dosierungsanweisungen und Applikationsformen sind anhand anderer Literaturstellen oder der Packungsbeilage auf ihre Richtigkeit zu überprüfen. Der Verlag übernimmt keine Gewähr. 